\title{
Role of reversibility in viral capsid growth: A paradigm for self-assembly
}

\author{
D. C. Rapaport* \\ Physics Department, Bar-Ilan University, Ramat-Gan 52900, Israel
}

(Dated: September 7, 2008)

\begin{abstract}
Self-assembly at submicroscopic scales is an important but little understood phenomenon. A prominent example is virus capsid growth, whose underlying behavior can be modeled using simple particles that assemble into polyhedral shells. Molecular dynamics simulation of shell formation in the presence of an atomistic solvent provides new insight into the self-assembly mechanism, notably that growth proceeds via a cascade of strongly reversible steps and, despite the large variety of possible intermediates, only a small fraction of highly bonded forms appear on the pathway.

PACS numbers: $81.16 . \mathrm{Fg}, 87.16 . \mathrm{Ka}, 02.70 . \mathrm{Ns}$
\end{abstract}

Structure formation in self-assembling molecular complexes is a particularly important process, both for nature and nanotechnology [1]. One very familiar example is the formation of viral capsids, the polyhedral shells of capsomer particles enclosing the genetic payload of spherical viruses [2, 3]. The ubiquity of icosahedral symmetry among spherical viruses, a design adopted by nature where shells are built by repeated use of just one or a small number of distinct capsomers, motivates a reduced description that avoids the molecular details of capsomer proteins; the fact that assembly also occurs in vitro [4, 5, 6] makes it an ideal candidate for simulation. If self-assembly is indeed governed by general organizational principles - with important consequences for both medicine and materials science - they ought to be accessible using simplified models.

There is little direct experimental evidence on the nature of assembly pathways, and since self-assembly implies a nonequilibrium state, where predictive theory is absent, simulation has a potentially important role. Molecular dynamics (MD) modeling of capsid selfassembly, based on simple structural models, was described in Refs. [7, 8]; the model particles retain just enough detail to ensure meaningful behavior, the two key features being an effective molecular shape formed out of soft spheres rigidly packed so particles fit together in a closed shell, and multiple interaction sites positioned to stabilize the correct final structure. The focus was on achieving assembly; pathways were not considered, and solvent was omitted to reduce computational cost.

The present paper describes MD simulations of selfassembling particles that incorporate an explicit atomistic solvent. Solvent presence aids cluster breakup without subassemblies needing to collide directly, curtails the ballistic nature of the particle motion ensuring conditions closer to equilibrium, serves as a heat bath to absorb energy released by bond formation, and maintains the dynamical correlations of a fluid medium. To anticipate the conclusions, self-assembly is found to consist of a cascade of reversible stages, with a strong preference

*Electronic address: rapaport@mail.biu.ac.il for low-energy intermediate states, eventually leading to a high yield of complete shells. Paradoxical though it may seem, reversibility is the key to efficient production. Even though the present focus is on icosahedral growth to lower the computational effort - these features of the behavior are likely to be entirely general.

An alternative particle-based, solvent-free simulation [9] treated quasi-rigid bodies made of hard spheres. Even simpler capsomer representations have been proposed with spherical particles instead of extended capsid shapes, and either directional interactions [10] whose range exceeds the particle size, or bonding energies determined by local neighborhood rules [11]; solvent is represented implicitly with stochastic forces. At the other extreme are the folded proteins of real capsomers; MD treatment of all-atom models [12] is limited to short trajectories to test stability of prebuilt shells. Capsid structure has also been studied by a variety of theoretical methods [13, 14, 15, 16, 17], and experiments have been interpreted using concentration kinetics [5, 18]; all avoid addressing the underlying discrete particle dynamics.

The simulations involve triangular particles based on the design and interactions of Ref. [8], that are expected to self-assemble into icosahedral shells. Particle shape is approximated by a rigid set of soft spheres with lateral faces inclined at $20.905^{\circ}$ to the normal, as shown in Fig. 1. There are four interaction sites on each lateral face that can bond to matching sites on adjacent particles; multiple sites help maintain correct alignment after bonding. The solvent atoms are identical to the particle spheres; all experience a soft-sphere repulsion whose parameters determine the dimensionless MD length and time units 19] used here. The attractive interaction between bondforming interaction sites is harmonic at distances below $r_{h}=0.3$ and inverse-square above $r_{h}$ with range $r_{a}=3$; the overall attraction strength is governed by a parameter $e$. The fact that particle size exceeds interaction range, although less so than in real capsomers, reduces the interaction of wrongly positioned particles.

MD methodology is described in Ref. [19]. The system consists of 125000 molecules in a cubic region with periodic boundaries; most are solvent atoms, but there are 1875 triangular particles (1.5\%), enough to produce 93 full shells; the mean number density is 0.2 . Although 


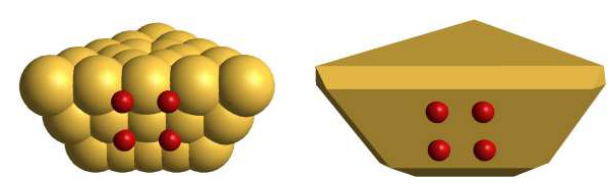

FIG. 1: (Color online) Model particle and the effective truncated pyramidal shape; small spheres denote interaction sites.

particle concentration is much higher than in experiment, the solvent presence is sufficient to ensure that diffusion tempers the otherwise ballistic particle motion. Runs of 60 million time steps are typically needed, at a rate of one million steps per day on a computer with dual $3.6 \mathrm{GHz}$ Intel processors; there are 200 steps per unit (MD) time. Particle mass, proportional to volume, is 21 times the solvent atom (with unit mass); having a much smaller ratio than in actual viruses leads to a reduced assembly timescale, accessible to MD, without qualitative change in behavior. Gradual heating by exothermal bond formation is suppressed by means of a thermostat that maintains a temperature of 0.667 , corresponding to unit mean kinetic energy. Runs are initialized by placing particles and solvent atoms on a lattice with random velocities; to avoid overlap, particles begin collapsed and expand to their final shape over the initial 5000 steps. No additional mechanisms are included, e.g., damping and partial shell breakup [8], to aid or regulate assembly.

The ability to produce complete shells is the principal characteristic of the method; since $e$ is the only parameter varied, coverage of the phase diagram is limited, but this proves adequate for demonstrating a variety of growth scenarios. Establishing shell completeness requires identifying bound clusters [19] and checking the bond network connectivity. There is a certain arbitrariness in the bond definition. Interaction sites are bonded when less than $0.6\left(=2 r_{h}\right)$ apart, a threshold that avoids transient bond breakage by thermal vibration. Particles are considered bonded if all four mutual site pairs are bonded, a state implying alignment; a weaker condition involving just a single site pair is used to search for loosely linked, misaligned clusters.

Figure 2 shows how the cluster size distributions, expressed in terms of mass fractions, evolve with time for particular $e$ values. The highest observed shell count, 83, occurs at $e=0.13$ and corresponds to a yield of $89 \%$. Growth curves have a sigmoidal shape; there is an initial lag until shells appear, followed by a period of rapidly increasing shell count that ends asymptotically. Further change, beyond the 60 million steps shown, is extremely slow. No oversized clusters are observed (although sufficiently large $e$ would lead to mutant forms).

There is a range of $e$ (exemplified by $e=0.13$ ) with efficient shell production, where small clusters grow to completion while maintaining an adequate monomer supply; the details depend on $e$. At lower $e(0.11)$ there is practically no growth due to minimal initiation. At higher $e(0.14$, and especially 0.15$)$ there is excessive early growth, resulting in too many monomers being incorporated into clusters prematurely, and preventing the appearance of complete shells until monomers are released by cluster breakup. The monomer disappearance rate increases with $e$, unlike the more complex $e$-dependent shell growth; for smaller $e$, a finite monomer fraction persists. While similar overall behavior is seen in reaction kinetics studies [5], provided nucleation is rate limited, MD needs no such restriction. Reproducibility is confirmed by repeating a run with a different initial state and obtaining similar results. Figure 3 provides a view of the $e=0.13$ system once 80 complete shells have formed.

Any tendency to form loosely linked clusters can be detected by relaxing the bonding criterion to require only a single interaction site pair. Cluster distributions are barely affected since bonds that persist eventually become part of a strong (four-pair) bond. Direct visualization reveals that adjacent partial shells can appear to be a single oversized cluster, but their weak binding allows them to separate with little impact on long-term growth. Shell stability is tested by extending a run that had already produced numerous closed shells, after reducing $e$ to a value where assembly yields only a few dimers. Residual small clusters promptly vanish, followed by the gradual disappearance of larger clusters; eventually just the original closed shells remain, together with the occasional dimer. This implies hysteresis [20], the survival of complete shells even when conditions become unfavorable.

Other aspects of cluster development require further analysis. While exhaustive shell histories are informative but not readily summarized, the statistics of event types as cluster membership changes provide insight into the process. Figure 4 shows event fractions and mean cluster lifetimes (lifetimes have broad distributions) for all sizes. The analysis considers particle configurations during the first 30 million steps of the run with the highest shell yield $(e=0.13)$, spaced every 2000 steps to minimize merged (e.g., two added monomers that appear as dimer addition) and missed (e.g., bonds that form and break) events; clusters present at the end are excluded. The principal event types are increases and decreases of unit size (including dimer breakup), and size increases $>1$; others less readily specified (e.g., size decreases $>1$ ) are grouped together (early in the run, there is slightly more dimer and trimer growth, and direct visualization reveals infrequent events such as pentamer bonding).

Reversibility plays a central role; for sizes $<19$ there is considerable dissociation, and for half the sizes breakup is even more likely than growth. The fact that growth events are only a fraction of the total enhances the assembly process by providing ample opportunity for the error correction needed to avoid kinetic traps. Reversibility also reflects a proximity to equilibrium, with a slight bias in favor of growth. The consequences of reversibility have also been considered [21] using reaction kinetics.

Insofar as lifetimes are concerned, pentamers live much longer than other small clusters; lifetime and growth 

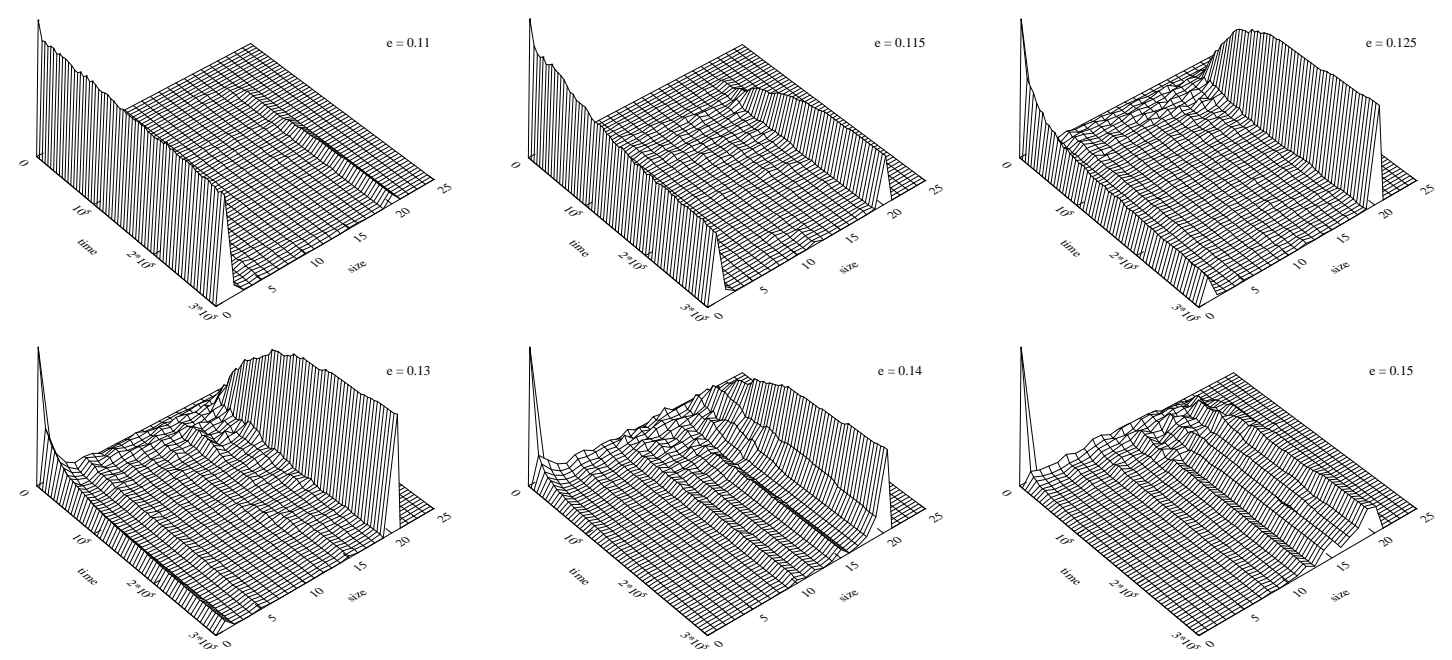

FIG. 2: Cluster size distributions as functions of time (MD units); the distributions, including monomers, are expressed as mass fractions, and $e$ (attraction strength) values are selected to show the different growth scenarios.

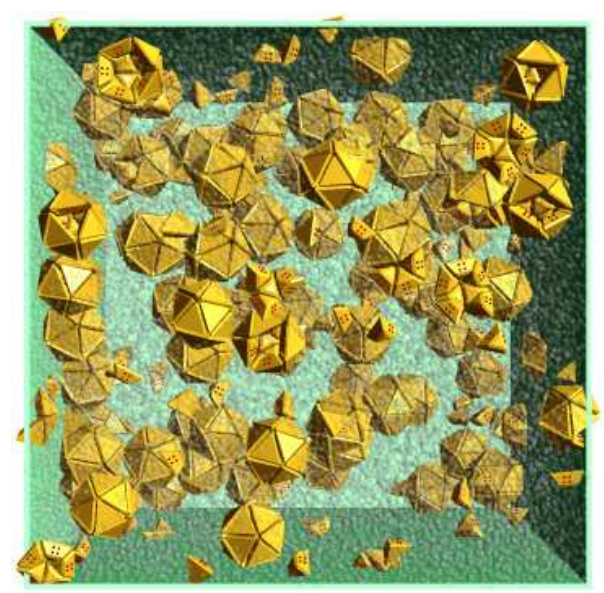

FIG. 3: (Color online) Snapshot of the $e=0.13$ system with 80 complete shells; solvent atoms are shown semitransparently, and there are visual artifacts (closed shells that appear open and particles protruding through walls) due to periodic boundaries.

probability appear correlated. Nearly complete shells live longest, but even at size 19 the wait until subsequent shell closure is only eight times the pentamer lifetime. Certain growth steps allow incoming particles to bond just along one face, a state favoring rapid disassembly (as in, e.g., hexamers), but even more stable intermediates can lose members. Other results (not shown) are that while growth probability and lifetime vary with $e$, the overall trends are similar, and that mean bond length ranges from 0.2 for dimers to 0.05 for closed shells.

The nature of the intermediate states along the growth pathways is an especially notable feature. Table I summarizes a series of measurements at half million step in-

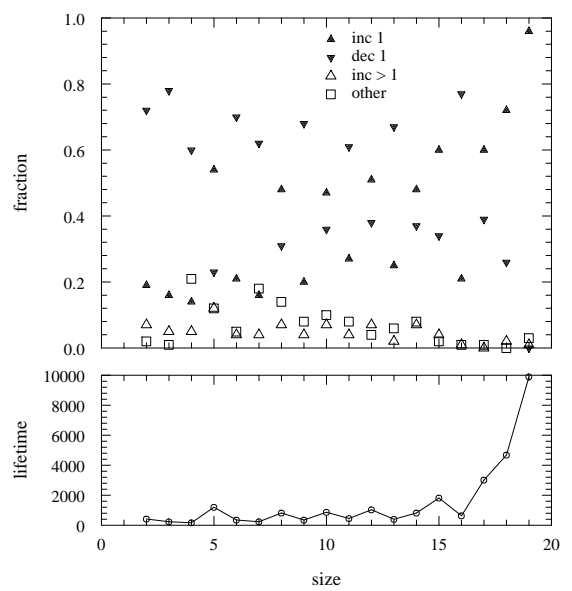

FIG. 4: Fraction of events producing unit size increases and decreases, increases greater than unity, and others; mean cluster lifetimes (MD units).

tervals, for $e=0.13$, in which clusters are grouped by bond count (other aspects of cluster geometry are not considered). There is a strong preference for maximally bonded (minimal energy) clusters, and all are within two bonds of maximum. Numbers of possible cluster realizations - equivalent to the distinct connected embeddings [22] of triangles in an icosahedron - are included for contrast. The majority are loosely bound and never observed as intermediate states; e.g., while $91.7 \%$ of clusters of size 12 are seen to adopt the unique 15 bond form, and the remainder have 14 or 13 bonds, none of the 446 possible realizations with fewer bonds are encountered. The effect of an imposed preference for maximally bonded intermediates has been studied with reaction kinetics [23]; in MD this property emerges naturally from the simulations. 
TABLE I: Intermediate states along the growth pathways: mean cluster fractions $(f)$ grouped by size $(s)$ and bonds $(b)$; numbers of distinct cluster realizations $(n)$ are included for comparison, with the final columns enumerating other realizations that are not observed (sizes with unique bond counts are omitted).

\begin{tabular}{|c|c|c|c|c|c|c|c|c|c|c|c|}
\hline \multirow[b]{2}{*}{$s$} & \multirow[b]{2}{*}{$b$} & \multirow[b]{2}{*}{$n$} & \multirow[b]{2}{*}{$f$} & \multirow[b]{2}{*}{$b$} & \multicolumn{3}{|c|}{ Observed } & \multirow[b]{2}{*}{$n$} & \multirow[b]{2}{*}{$f$} & \multicolumn{2}{|c|}{ Others } \\
\hline & & & & & $n$ & $f$ & $b$ & & & $b$ & $n$ \\
\hline 5 & 5: & 1 & 0.948 & 4: & 5 & 0.052 & & & & & \\
\hline 6 & $6:$ & 1 & 0.953 & 5: & 13 & 0.047 & & & & & \\
\hline 7 & 7: & 4 & 0.979 & $6:$ & 22 & 0.021 & & & & & \\
\hline 8 & 9: & 1 & 0.851 & 8: & 11 & 0.140 & 7: & 46 & 0.009 & & \\
\hline 9 & 10: & 3 & 0.938 & 9: & 27 & 0.062 & & & & 8: & 79 \\
\hline 10 & 12: & 1 & 0.808 & 11: & 13 & 0.166 & 10: & 60 & 0.026 & 9: & 151 \\
\hline 11 & 13: & 3 & 0.931 & 12: & 28 & 0.069 & & & & 11-10: & 328 \\
\hline 12 & 15: & 1 & 0.917 & 14: & 11 & 0.073 & 13: & 74 & 0.010 & 12-11: & 446 \\
\hline 13 & 16: & 4 & 0.876 & 15: & 31 & 0.105 & 14: & 142 & 0.019 & 13-12: & 372 \\
\hline 14 & 18: & 1 & 0.802 & 17: & 15 & 0.198 & & & & 16-13: & 417 \\
\hline 15 & 20: & 1 & 0.825 & 19: & 5 & 0.146 & 18: & 38 & 0.029 & 17-15: & 170 \\
\hline 16 & 21: & 4 & 0.915 & 20: & 19 & 0.068 & 19: & 38 & 0.017 & 18: & 28 \\
\hline 17 & 23: & 1 & 0.923 & 22: & 7 & 0.077 & & & & 21: & 12 \\
\hline 18 & 25: & 1 & 0.888 & $24:$ & 5 & 0.112 & & & & & \\
\hline
\end{tabular}

[1] G. M. Whitesides and B. Grzybowski, Science 295, 2418 (2002).

[2] F. H. C. Crick and J. D. Watson, Nature (Lond.) 177, 473 (1956).

[3] D. L. D. Caspar and A. Klug, Cold Spring Harbor Symp. Quant. Biol. 27, 1 (1962).

[4] P. E. Prevelige, D. Thomas, and J. King, Biophys. J. 64, 824 (1993).

[5] A. Zlotnick, J. M. Johnson, P. W. Wingfield, S. J. Stahl, and D. Endres, Biochemistry 38, 14644 (1999).

[6] G. L. Casini, D. Graham, D. Heine, R. L. Garcea, and D. T. Wu, Virology 325, 320 (2004).

[7] D. C. Rapaport, J. E. Johnson, and J. Skolnick, Comp. Phys. Comm. 121, 231 (1999).

[8] D. C. Rapaport, Phys. Rev. E 70, 051905 (2004).

[9] H. D. Nguyen, V. S. Reddy, and C. L. Brooks III, Nano Letters 7, 338 (2007).

[10] M. F. Hagan and D. Chandler, Biophys. J. 91, 42 (2006).

[11] R. Schwartz, P. W. Shor, P. E. Prevelige, and B. Berger, Biophys. J. 75, 2626 (1998).

[12] P. L. Freddolino, A. S. Arkhipov, S. B. Larson, A. McPherson, and K. Schulten, Structure 14, 437
In conclusion, self-assembly at submicroscopic scales, where atomistic effects become important, is very different from inherently unidirectional macroscopic assembly. MD simulation reveals reversibility along the assembly pathway, with dissociation often more likely than growth. Reversibility diminishes the significance of kinetic traps because escape is accomplished by dissociation. The coexistence of reversibility and a high error-free yield is a result likely to have important implications for understanding supramolecular assembly in general and capsid formation in particular.

The author is grateful to A. Zlotnick for helpful discussion.
(2006).

[13] J. Lidmar, L. Mirny, and D. R. Nelson, Phys. Rev. E 68, 051910 (2003).

[14] R. Twarock, J. Theor. Biol. 226, 477 (2004).

[15] R. Zandi, D. Reguera, R. F. Bruinsma, W. M. Gelbart, and J. Rudnick, Proc. Natl. Acad. Sci. USA 101, 15556 (2004).

[16] S. D. Hicks and C. L. Henley, Phys. Rev. E 74, 031912 (2006).

[17] M. Hemberg, S. N. Yaliraki, and M. Barahona, Biophys. J. 90, 3029 (2006).

[18] P. van der Schoot and R. Zandi, Phys. Biol. 4, 296 (2007).

[19] D. C. Rapaport, The Art of Molecular Dynamics Simulation (Cambridge University Press, Cambridge, 2004), 2nd ed.

[20] S. Singh and A. Zlotnick, J. Biol. Chem. 278, 18249 (2003).

[21] A. Zlotnick, J. Mol. Biol. 241, 59 (1994).

[22] D. C. Rapaport, Computer Phys. Repts. 5, 265 (1987).

[23] D. Endres, M. Miyahara, P. Moisant, and A. Zlotnick, Protein Sci. 14, 1518 (2005). 